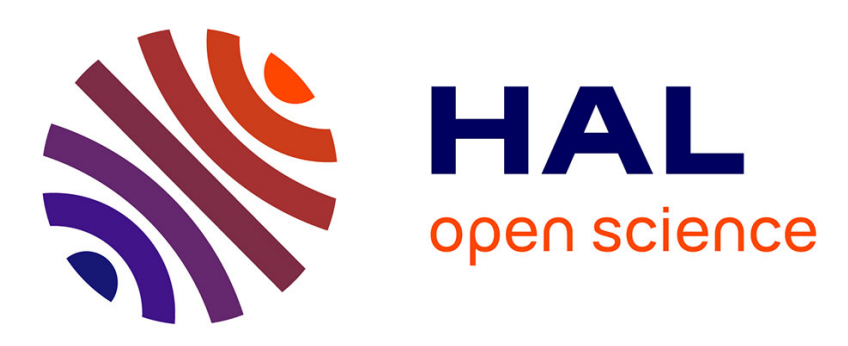

\title{
Multi-resolution localization method based on time reversal and simulated annealing algorithm
}

Nadia Aloui, Kosai Raoof, Ammar Bouallegue, Stephane Letourneur, Sonia Zaibi

\section{- To cite this version:}

Nadia Aloui, Kosai Raoof, Ammar Bouallegue, Stephane Letourneur, Sonia Zaibi. Multi-resolution localization method based on time reversal and simulated annealing algorithm. IPIN 2013 - 4th International Conference on Indoor Positioning and Indoor Navigation, Oct 2013, Montbéliard, France. pp.1 - 9, 10.1109/IPIN.2013.6817865 . hal-01099468

\section{HAL Id: hal-01099468 https://hal.science/hal-01099468}

Submitted on 3 Jan 2015

HAL is a multi-disciplinary open access archive for the deposit and dissemination of scientific research documents, whether they are published or not. The documents may come from teaching and research institutions in France or abroad, or from public or private research centers.
L'archive ouverte pluridisciplinaire $\mathbf{H A L}$, est destinée au dépôt et à la diffusion de documents scientifiques de niveau recherche, publiés ou non, émanant des établissements d'enseignement et de recherche français ou étrangers, des laboratoires publics ou privés. 


\title{
Multi-resolution Localization Method Based On Time Reversal And Simulated Annealing Algorithm
}

\author{
Nadia ALOUI*†, Kosai RAOOF ${ }^{\ddagger}$, Ammar BOUALLEGUE ${ }^{\dagger}$, Stephane LETOURNEUR ${ }^{\ddagger}$ and Sonia ZAIBI ${ }^{\dagger}$ \\ ${ }^{*}$ GIPSA-lab, CNRS UMR5216, Universit Joseph Fourier, Grenoble, France \\ ${ }^{\dagger}$ Universite de Tunis El Manar, Ecole Nationale dIngenieurs de Tunis, \\ LR-99-ES21 Laboratoire de Systemes de Communications, 1002, Tunis, Tunisie \\ ${ }^{\ddagger}$ LAUM Laboratory UMR CNRS 6613, University of Maine, Le Mans, France \\ Email: nadia.aloui@gipsa-lab.grenoble-inp.fr \\ Email:kosai.raoof@univ-lemans.fr
}

\begin{abstract}
In this paper, we propose a novel acoustic indoor localization system that is based on time reversal. The system estimates the target position with different precision. First, it uses a low frequency signal in order to localize the source in the whole area within coarse precision. Then, it switches to high frequency signal to estimate the target location in a reduced area within a fine precision.

The system performance have been evaluated by simulations and by experiments conducted in a practical training room in the National School of Engineers of Le Mans. Results have shown that the system has subcentimeter accuracy.
\end{abstract}

Keywords : Time reversal, coarse precision, fine precision, simulated annealing algorithm.

\section{INTRODUCTION}

Indoor location estimation has useful applications in our everyday life. For example, in hospitals, based on location information, doctors can reach their patients rapidly. In an enterprise, employees can be located and property can be controlled to avoid their stealing. In a supermarket, a user can locate the promotional items close to him. He can also search for an item and estimate its position as well as his own position.

Outdoor positioning systems such as the Global Positioning System [1] afford good estimation accuracy in outdoor environments. However, they fail indoors.

In this work, we describe a novel acoustic indoor localization system that is based on time reversal. This method allows an efficient focus of an acoustic signal regardless of the position of the initial source and regardless of the heterogeneity of the propagation medium. The system aims to locate the target with different precision.

In order to validate this concept, two carrier frequencies scrambled with Gold sequence are emitted. The received signals are time reversed and reemitted simultaneously by four transducers. A receiver will demodulate and descramble the two signals in function of the desired precision. First, it uses the low frequency descrambled signal to estimate the source position in the whole area within a coarse precision. It

978-1-4673-1954-6/12/\$31.00 2012 IEEE moves in the area space according to the simulated annealing algorithm in order to search for the position that maximizes the descrambled signal. Then, it can switch to high frequency signal in order to localize the source in a reduced area within a fine precision, based on the position estimated in the first step.

The following section gives a brief overview of indoor location systems. Section III describes the time reversal technique. The proposed system is described in section IV. Section V presents the simulated annealing algorithm. System performance is given in section VI. The last section concludes the paper.

\section{RELATED WORK}

Several indoor location systems have been developed in order to overcome the failure of outdoor positioning systems to work in indoor environments. These systems employ various location techniques such that triangulation and fingerprinting technique.

Triangulation requires the line of sight between transmitter and receiver. It comprises angulation and lateration. In order to calculate the 2D-location of the target, the lateration needs at least three measured distances between the target and references of known positions, while, the angulation needs the measured angles between the target and at least two known locations. Fingerprinting technique comprises two phases: offline and on-line stages. In the first phase, a set of sampling locations is selected in the target environment, then, a location dependent signal parameter is extracted at each location to define the location signature. In the second phase, the target signature is compared with signatures in the previously built database and the position is estimated through pattern matching algorithms.

RADAR [2] is an example of fingerprint-based location systems. It uses the radio frequency technology and deploys three base stations. Two approaches for constructing the fingerprint database have been considered. The first is the empirical method where the Signal Strength data are gathered during the off-line phase. The second approach is signal propagation modeling where a set of theoretically-computed signal strength data are generated. Results have shown that the empirical 
method is more accurate than the propagation method and the system provides an accuracy of $3 \mathrm{~m}$ with $50 \%$ precision. Another fingerprint-based location system is described in [3]. The system employs audible signals that consist of Binary Phase Shift Keying modulated Gold sequences and adopts the Time of arrival of path of maximum amplitude as a fingerprint. Three sources at known locations are deployed and the target location is accomplished through the local linear estimator. The system provides an accuracy of $8.5 \mathrm{~cm}$ with $80 \%$ precision. On the other hand, Active bat [4] and the cricket systems [5] use triangulation technique. The Active bat employs ultrasound waves and provides 3D-position and orientation information for tags taken by users. The system works as follows: First, the tag periodically broadcasts a short pulse of ultrasound. A matrix of ceiling fixed receivers at known locations receives the short pulse. Then, the TOA of ultrasonic waves between the tag and the receivers are measured and the distances are inferred. Three distances (between the tag and three receivers) are required to measure the 3D-position of the tag. The system provides an accuracy of about $3 \mathrm{~cm}$ for $95 \%$ of the measurements. However, it requires deploying a large number of sensors on the ceiling in each room, which is a timeconsuming task.

In the cricket system, the ultrasound emitters are fixed on ceiling or walls at known positions. A receiver is attached on each target. The target location is estimated based on TOA measurements and triangulation technique. The ultrasound emitters also transmit RF messages for synchronization of TOA measurement. The system provides a position accuracy of $10 \mathrm{~cm}$ and an orientation accuracy of $3^{\circ}$. The cricket system uses less number of emitters mounted on ceiling than that the number of receivers used by the Bat system. However, since the target receives US and RF signals at the same time the power consumption is higher.

Authors in [6] have proposed an indoor localization system that employs CDMA signals and uses the lateration location technique. The TOA of the acoustic signals are estimated as the instant of the peak correlation between the received signal and the emitted signal. The distances between the speakers and the localized object are then deduced from the obtained TOA. The system provides location estimates of better than $2 \mathrm{~cm}$ accuracy with $99 \%$ precision.

On the other hand, time reversal has been applied in localization purpose. In [7], a localization system based on time reversal is described. The system aims to locate a node in wireless sensor network. The node that wants to locate itself sends a request signal to beacon nodes around and records the starting time. The beacon nodes time reverse the received signal and emits it in the medium. When the unknown node receives the focus signal, it records the finishing time. The time differences are computed and the distances are inferred. Then, the node coordinates are estimated based on a certain algorithm. Since the time reversal compresses the multipath channel, the instant signal-to-noise ratio (SNR) is improved and the localization error is reduced. Simulation results have shown that the time reversal scheme is better than the normal scheme when the nodes number is less than five.

The work in [8] deals with port security and aims to provide protection against underwater threats. The main idea is to use acoustic noise radiated by a hostile diver to focus the acoustic signal back to a diver. The system can be described in the following steps: The signal generated by the swimmer is first recorded by the hydrophones of time reversal acoustic (TRA) system and sent to a TRA electronic unit for processing. Second, the recorded signals are filtered, amplified and time reversed. Third, the resulted signals are simultaneously radiated by the underwater hydrophones. The radiated signals are automatically focused to the swimmer (e.g. the initial point of radiation). Simulations have shown that a TRA of 20 transmitters with a peak acoustic power of $100 \mathrm{~W}$ is able to disorient and divert the swimmer away, when the distance between the diver and transmitters is about $200 \mathrm{~m}$. With 5 transducers, the system can disorient a swimmer at $50 \mathrm{~m}$ from transducers.

The main contribution of our work is to locate the target with different precision based on time reversal. A description of Time reversal is given next.

\section{FORMALISM OF TIME REVERSAL}

Time reversal has many applications comprising underwater acoustics, telecommunications, room acoustics and ultrasound medical imaging and therapy [9]. It is based on the invariance of wave propagation equation if time is reversed. In a nondissipative medium, the acoustic wave equation is invariant under a time-reversal operation. Indeed, it comprises a secondorder-time-derivative operator. Therefore, to any wave $p(r, t)$ diverging from the source corresponds a wave $p(r,-t)$ converging to the source. Based on this property and on the principle of Helmoltz-Kirchoff, D.Cassereau and M.Fink [10] have developed the concept of the cavity of Time reversal. Indeed, they have demonstrated that by exploiting the Helmoltz-Kirchoff principle, the operation of time reversal does no longer consist in reversing the acoustic wave in every point of the considered volume, but only the wave and its normal derivative in the surface bounding the volume. The time reversal operation can then be described in two phases: the reception phase and the emission phase. In the first phase, a source situated inside the cavity emits an acoustic pulse in the medium. The latter must be non-dissipative but can be heterogeneous. The generated spherical wave is reflected, diffracted and diffused in the heterogeneous environment. When the wave reaches the surface $S$ bounding the volume, the wave and its normal derivative are recorded by the transducers, and then stored in memory. During the second phase, the time reversed fields are re-emitted by every transducer in the medium. Didier Cassereau and Mathias Fink have demonstrated that the created field is equal to the initially emitted wave but time reversed [10]. This wave converges to the initial source. The authors have also demonstrated that the size of the focal spot, defined as the width at half-height of the zone where the energy is concentrated, is limited by the diffraction phenomenon. Its dimension is about the half of the wavelength of the wave.

Theoretically, the concept of Time Reversal Cavity allows the sound waves to propagate back through the medium. Nevertheless, in practice, this cavity appears to be difficult 
to achieve because it requires a large number of transducers. It can then be replaced by a non-closed surface, called the time reversal mirror. The latter does not recreate the field as accurately as the time reversal cavity does but it allows an accurate and an efficient focus of the recorded wave on acoustic sources.

From signal processing point of view, assuming a linear time-invariant system, the signal received at position $s_{i}$, after the emission of signal $G(t)$ by a source situated at $s$ can be written as:

$$
r(t)=G(t) \otimes h\left(s, s_{i}, t\right)
$$

We consider that every elementary source, $s_{i}$, of the cavity emits the signal that it has received but in inverse chronology:

$$
r(-t)=G(-t) \otimes h\left(s, s_{i},-t\right)
$$

Where $h\left(s, s_{i}, t\right)$ denotes the impulse response between $s$ and $s_{i}$. The signal received at any point $s s$ can therefore be written as:

$$
r(t)=\sum_{i} G(-t) \otimes h\left(s, s_{i},-t\right) \otimes h\left(s_{i}, s s, t\right)
$$

At the position $s s=s$ which corresponds to the position of the initial source we obtain

$$
r(t)=\sum_{i} G(-t) \otimes h\left(s, s_{i},-t\right) \otimes h\left(s_{i}, s, t\right)
$$

Time reversal is then a matched filter in the sense of signal processing. During the first phase of time reversal, the initially emitted signal is filtered by the propagation medium. Every spectral component of the impulse responses has particular amplitude and phase. Time reversal returns in the medium each impulse response without changing the relative amplitudes of the various frequency components so that they are exactly adapted to the environment. It also offsets their phases so that they summon all consistently at a point and at a given time.

In the following, we describe our proposed method.

\section{MUlti-RESOlUtion LOCATION METHOD}

The proposed method aims to localize a source with high localization precision. The main idea is to send two signals simultaneously with different wavelengths. The receiver searches for the source in the whole zone of interest using the low frequency range. Then, it switches to high frequency range to localize the source in a reduced area based on the previously estimated position (see figure 1).

The method can be described in the following steps:

- A source, at position $s$, emits two carrier frequencies scrambled with Gold sequence $g(t)$ :

$$
\begin{array}{r}
G(t)=g(t) \times \sin \left(2 \times \pi \times f_{1} \times t\right)+ \\
g(t) \times \sin \left(2 \times \pi \times f_{2} \times t\right)
\end{array}
$$

$f_{1}$ and $f_{2}$ are respectively the low and high carrier frequencies.

- A transducer $i$, at position $s_{i}$, receives the signal

$$
y_{i}(t)=G(t) \otimes h_{i}\left(s, s_{i}, t\right)+n_{i}(t)
$$

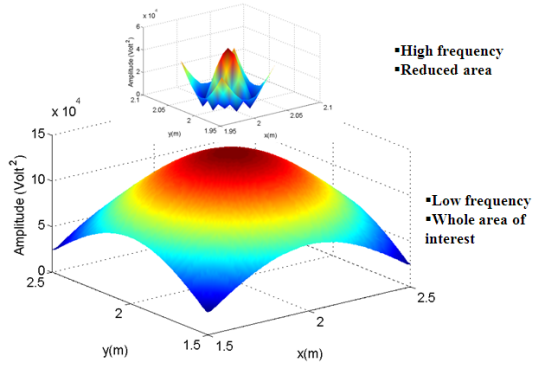

Fig. 1: Multi-resolution Location Method

Where $n_{i}(t)$ denotes the channel noise at the transducer $i$ and $h_{i}\left(s, s_{i}, t\right)$ denotes the channel impulse response between the source and the transducer $i$ and is given by:

$$
h_{i}\left(s, s_{i}, t\right)=\sum_{l=1} \alpha_{l, i} \times \delta\left(t-\tau_{l, i}\right)
$$

$\alpha_{l, i}$ and $\tau_{l, i}$ are respectively the attenuation and the delay of the path $l$.

- The received signals are time reversed and re-emitted simultaneously and continuously by four transducers

$$
y_{i}(-t)=G(-t) \otimes h_{i}\left(s, s_{i},-t\right)+n_{i}(-t) ; i=1, \ldots, 4
$$

- A microphone, at position $s s$, that wants to locate the source, receives the aggregate signal:

$$
Y(t)=\sum_{i=1}^{4} y_{i}(-t) \otimes h_{i}^{\prime}\left(s_{i}, s s, t\right)+b(t)
$$

Where $b(t)$ denotes the channel noise at the microphone and $h_{i}^{\prime}\left(s_{i}, s s, t\right)$ denotes the channel impulse response between the transducer $i$ and the microphone and is given by:

$$
h_{i}^{\prime}\left(s_{i}, s s, t\right)=\sum_{k=1} \beta_{k, i} \times \delta\left(t-\theta_{k, i}\right)
$$

$\beta_{k, i}$ and $\theta_{k, i}$ are respectively the attenuation and the delay of the path $k$.

- The receiver demodulates and descrambles the two signals in function of the desired precision (figure 2).

- First, it uses the low frequency descrambled signal $c_{L F}(s, s s, t)$ in order to estimate the source position in the whole area within a coarse precision.

- It computes the low frequency spatial correlation diagram given by:

$$
d_{L F}(s s)=\max _{t}\left(\left|c_{L F}(s, s s, t)\right|\right)
$$


Where

$$
\begin{aligned}
&\left|c_{L F}(s, s s, t)\right|^{2}=\left(\sum_{i=1}^{4} \sum_{l, k} \alpha_{l, i} \times \beta_{k, i}\right. \\
& \times \frac{\cos \left(2 \times \pi \times f_{1} \times\left(\tau_{l, i}-\theta_{k, i}\right)\right)}{2} \\
&\left.\times A_{g}\left(t-\tau_{l, i}+\theta_{k, i}\right)+v(t)\right)^{2}+\left(\sum_{i=1}^{4} \sum_{l, k} \alpha_{l, i} \times \beta_{k, i}\right. \\
& \times \frac{\sin \left(2 \times \pi \times f_{1} \times\left(\tau_{l, i}-\theta_{k, i}\right)\right)}{2} \\
&\left.\times A_{g}\left(t-\tau_{l, i}+\theta_{k, i}\right)+w(t)\right)^{2}
\end{aligned}
$$

$A_{g}$ denotes the autocorrelation function of the Gold sequence $g(t)$ and $v(t)$ and $w(t)$ the noise components after being demodulated and correlated with $g(-t)$.

- The receiver moves in the interested area, according to an optimization algorithm, in order to search for the position that maximizes $d_{L F}(s s)$.

- Second, it switches to high frequency signal in order to localize the source in a reduced area within a fine precision. So, it computes the high frequency descrambled signal $c_{H F}(s, s s, t)$ and the high frequency spatial correlation diagram $d_{H F}(s s)$ :

$$
d_{H F}(s s)=\max _{t}\left(\left|c_{H F}(s, s s, t)\right|\right)
$$

The reduced area is a square centered on the position obtained with the low frequency signal.

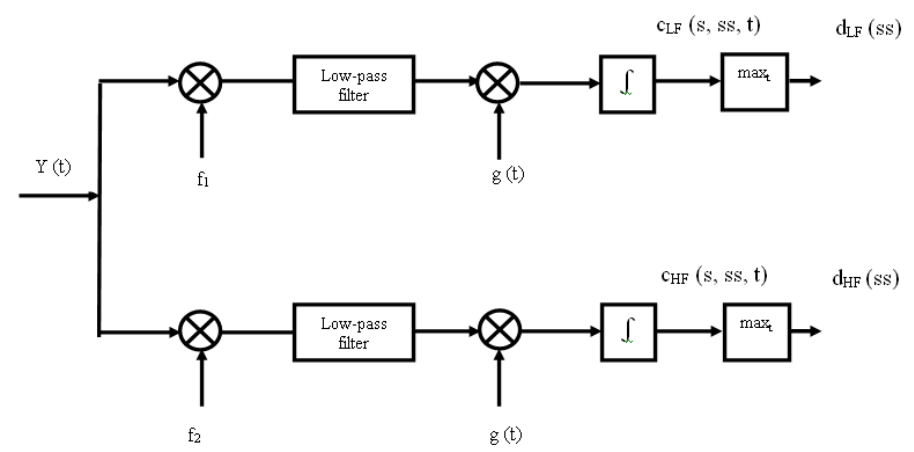

Fig. 2: The signal processing at the receiver

The terms $\cos \left(2 \times \pi \times f \times\left(\tau_{l, i}-\theta_{k, i}\right)\right)$ and $\sin (2 \times \pi \times$ $\left.f \times\left(\tau_{l, i}-\theta_{k, i}\right)\right)$ that appear in equation (12) control the shape of the correlation diagram. Indeed, for low value of frequency $f$, these terms vary slowly with the receiver position $\left(\theta_{k, i}\right)$ which induces a flat peak, while, for high frequency, they vary fast, generating then sharp peaks. For the last case, the area of interest may present pseudo-peaks in the neighborhood of the source which may cause errors in the estimation of the source position (see figure 3 ).

The flatness of the zone and the appearance of pseudo peaks make difficult the detection of the source position respectively

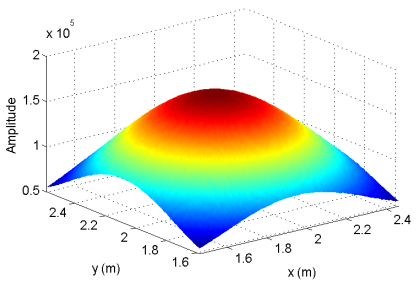

(a) low frequency

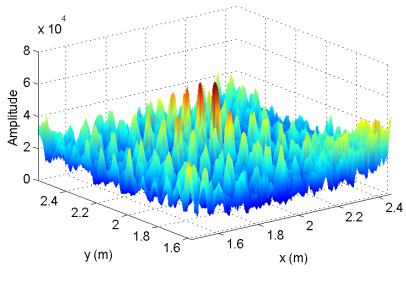

(b) high frequency
Fig. 3: Spatial correlation diagrams

for low and high frequency range. However, taking advantage of both ranges allows high localization accuracy.

For the receiver displacement, we have adopted the simulated annealing algorithm. The next section gives details about this algorithm

\section{Simulated AnNEALING ALGORITHM}

Simulated annealing is a generic probabilistic metaheuristic random technique for finding global extremums to large optimization problems. It is inspired from annealing in metallurgy, which involves heating material to above its critical temperature, maintaining a suitable temperature, and then cooling. These cycles induce the minimization of the material energy. By analogy with the physical process, the objective function is the system energy and the system temperature is used as a control parameter. The advantage of the annealing algorithm compared to other methods is its ability to avoid local extremums. Indeed, the algorithm accepts not only changes that optimize the objective function but also some changes that degrade it. The acceptance of good solution (i.e., a solution that improves the objective function) tends to seek the optimum in the vicinity of the starting solution. The acceptance of a bad solution then allows the exploration of a larger part of the solution space and tends to avoid locking too fast in finding a local optimum. The bad solutions are accepted with a probability:

$$
\exp \left(\frac{-\Delta f}{T(t)}\right)
$$

where $\Delta f$ is the variation of the objective function $f$ and $T$ is a control parameter corresponding to the system temperature in the physical process.

The figure 4 shows the structure of the simulated annealing algorithm. Assuming a minimization problem, the steps of simulated annealing algorithm are the following:

1) Choose an initial solution $x_{0}$ and compute $f\left(x_{0}\right)$

2) Put $i=1$

3) Choose a neighbor $x_{n e w}$ of $x_{i-1}$ at random

4) Compute $\Delta f=f\left(x_{n e w}\right)-f\left(x_{i-1}\right)$

a) If $\Delta f \leq 0$, then $x_{i}=x_{n e w}$

b) If $\Delta f>0$, then

- $x_{i}=x_{n e w}$ with probability $\exp \left(\frac{-\Delta f}{T(t)}\right)$

- $x_{i}=x_{i-1}$ otherwise. 
5) Increment $i$ by one and repeat the process from point 3

Generally, the temperature is reduced each time a certain number of iterations is performed. In this paper, we have adopted the following rule for temperature decrease:

$$
T_{i}=T_{0} \times \alpha^{i}
$$

Where $i \in\{0,1, \ldots\}$ and $0.9<\alpha<1$.

The temperature plays an important role. At high temperatures, the probability of acceptance is close to 1 . The system is free to move in the space of solutions by choosing solutions not necessarily minimizing the energy of the system. At low temperatures, changes decreasing the energy of the system are chosen, but others may be accepted, thus preventing the algorithm from falling into a local minimum.

The simulated annealing is adopted, in this paper, in order to avoid the little local optimums that may appear in the low frequency spatial correlation diagram. Simulations have proven that, for high frequency signal, the simulated annealing needs too much time to escape pseudo peaks. For this case, the receiver will compute the $d_{H F}(s s)$ at every position of the reduced area and saves the maximum value of $d_{H F}(s s)$ and the corresponding position. The estimate source position corresponds to the last saved position.

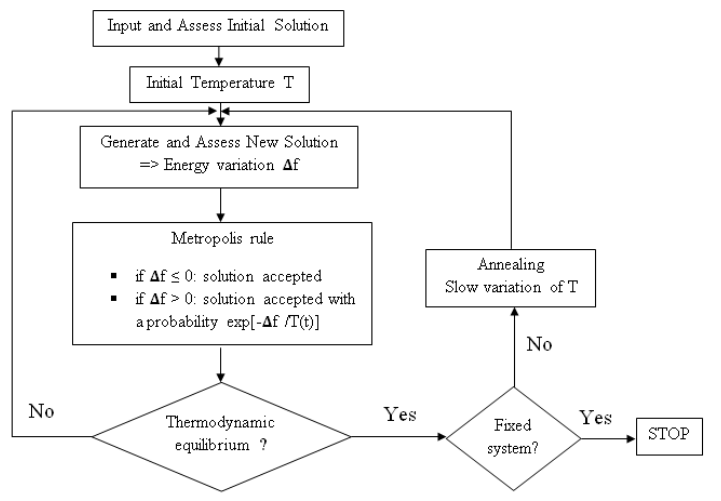

Fig. 4: Structure of the simulated annealing algorithm

\section{Evaluation}

The system performance are evaluated through simulations and experiments. Performance are given in terms of precision versus accuracy plots and in terms of precision distributions in the work area. The accuracy is referred to the highest tolerated error for a measure to be regarded as successful. And the precision is the ratio of the measurements with errors equal to or less than the accuracy.

\section{A. Simulations}

Performance of the proposed method is first evaluated through simulations.

We have chosen the channel model given by Allen and Berkley [11] in order to emulate the acoustic reflections in an empty room. This model is based on image technique where each image of the source contributes only a pure impulse of known strength and delay. The impulse response for a source situated at $r_{s}=\left(x_{s}, y_{s}, z_{s}\right)$ and a microphone placed at $r=(x, y, z)$ can then be written as:

$$
\begin{aligned}
& h\left(r_{s}, r, t\right)=\sum_{p \in P} \sum_{m \in M} \beta_{x_{1}}^{\left|m_{x}-q\right|} \beta_{x_{2}}^{\left|m_{x}\right|} \\
& \beta_{y_{1}}^{\left|m_{y}-j\right|} \beta_{y_{2}}^{\left|m_{y}\right|} \beta_{z_{1}}^{\left|m_{z}-k\right|} \beta_{z_{2}}^{\left|m_{z}\right|} \frac{\delta(t-\tau)}{4 \pi d}
\end{aligned}
$$

$d$ denotes the distance between the microphone and a source image, $\tau$ the time delay of arrival of the reflected sound ray corresponding to a source image and $\beta_{x_{1}}, \beta_{x_{2}}, \beta_{y_{1}}, \beta_{y_{2}}, \beta_{z_{1}}$ and $\beta_{z_{2}}$ the reflection coefficients of the six walls.

We have placed four transducers at positions $(0.2 m, 2 m, 2 m), \quad(2 m, 0.2 m, 2 m), \quad(2 m, 3.8 m, 2 m) \quad$ and $(3.8 m, 2 m, 2 m)$ in a room of dimensions $4 m \times 4 m \times 2.2 m$. The Gold sequence $g(t)$ emitted by the source is of length 127 chips and the carrier frequencies are fixed to $200 \mathrm{~Hz}$ and $4 \mathrm{kHz}$. The reflection coefficients of the six walls are set to 0.7 and reflections till order 12 are considered.

We place randomly nine sources in the room as shown in figure 5. We assume that the receiver that wants to estimate the source position is situated in a square centered on the source and of dimensions $d \times d \mathrm{~m}^{2}$. First, the receiver uses the low frequency descrambled signal in order to estimate the target position in the whole area within a coarse precision, then, it moves in the interested area according to the simulated annealing algorithm with a step $p$ of maximum $1 \mathrm{~cm}$ in $x$ direction and $1 \mathrm{~cm}$ in $y$ direction. Second, it switches to high frequency descrambled signal in order to estimate the source position in the reduced area within a fine precision.

The parameters of the simulated annealing algorithm are adjusted empirically: the parameter $\alpha$ and the initial temperatures $T$ are set to 0.98 and 100 respectively. At fine resolution, the side of the reduced square is set to $16 \mathrm{~cm}$.

500 positions of the receiver are randomly generated in the whole search area.

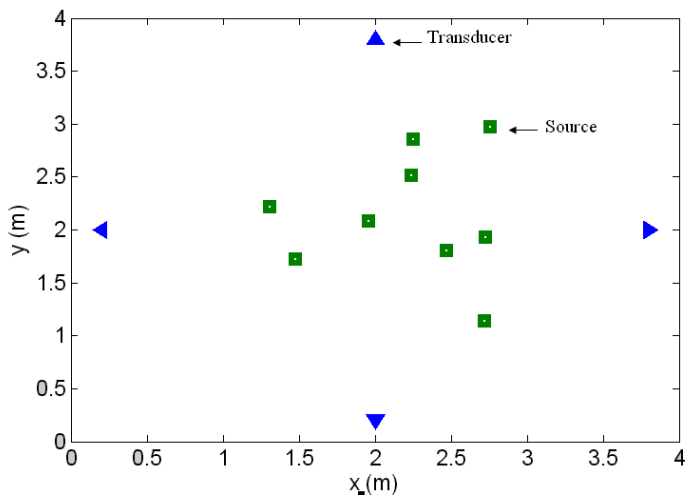

Fig. 5: The layout of the experiment setup

Precision versus accuracy for $d=1 \mathrm{~m}$ is plotted in figure 6 . 
TABLE I: Precision distribution for $1 \mathrm{~cm}$ accuracy, $d=1 m, T \in\{0,100\}$

\begin{tabular}{|c|c|c|c|}
\hline Source number & Positions & $T=0$ & $T=100$ \\
\hline 1 & $(1.96,2.08,1.5)$ & 96 & 100 \\
\hline 2 & $(2.73,1.93,1.5)$ & 81 & 93 \\
\hline 3 & $(2.47,1.8,1.5)$ & 97.2 & 98.2 \\
\hline 4 & $(2.72,1.14,1.5)$ & 96.8 & 97.8 \\
\hline 5 & $(2.24,2.51,1.5)$ & 92 & 96.6 \\
\hline 6 & $(1.48,1.72,1.5)$ & 79.2 & 91 \\
\hline 7 & $(2.25,2.85,1.5)$ & 81.4 & 94.8 \\
\hline 8 & $(2.76,2.97,1.5)$ & 92.4 & 94.8 \\
\hline 9 & $(1.31,2.22,1.5)$ & 87 & 93.20 \\
\hline
\end{tabular}

The system provides an accuracy of $0 \mathrm{~cm}$ within $74 \%$ precision and $1 \mathrm{~cm}$ within $95 \%$ precision. Precision per position for accuracy of $1 \mathrm{~cm}$ is depicted in figure 7 and illustrated in table I. The best precision is obtained at the source that is near to the center of the room.

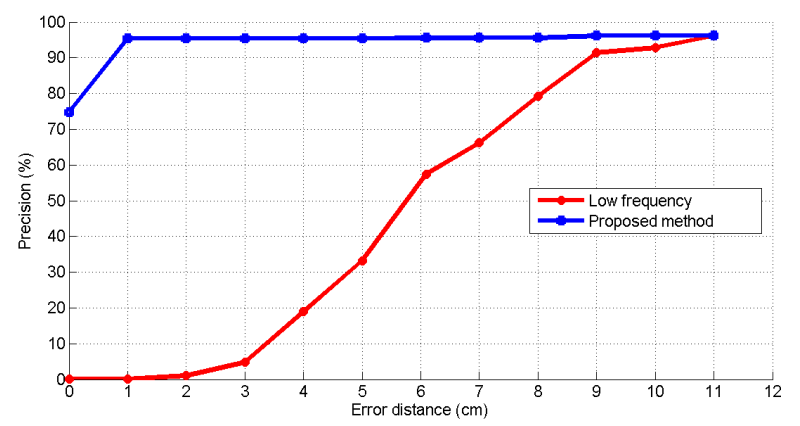

Fig. 6: Precision versus accuracy, $d=1 \mathrm{~m}$

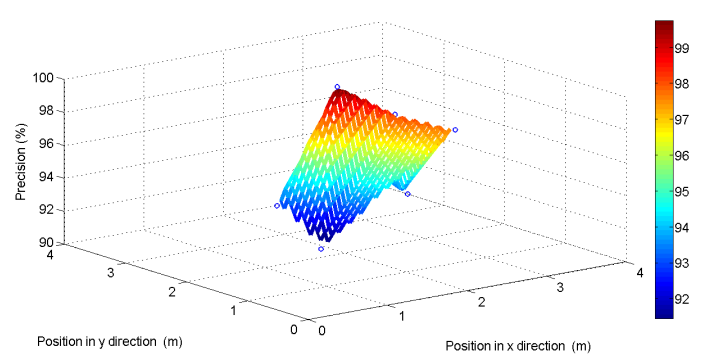

Fig. 7: Precision distribution for $1 \mathrm{~cm}$ accuracy, $d=1 \mathrm{~m}$

Then, we compare the system performance for $d=1 \mathrm{~m}$ when assigning to the initial temperature $T$ the values 0 and 100. At zero temperature, the simumated annealing algorithm does stochastic descent. Results shown in figure 8 indicate that precision obtained with $T=100$ is better than that obtained with $T=0$. Indeed, for $T=0$, no bad solution is accepted and therefore the receiver cannot reach the global optimum. However, for $T=100$, the acceptance of bad solution allows the receiver to escape local optimum and then finds the global one.

Moreover, we study the effect of the variation of the square width $d$ on the system performance. As shown in table II,we

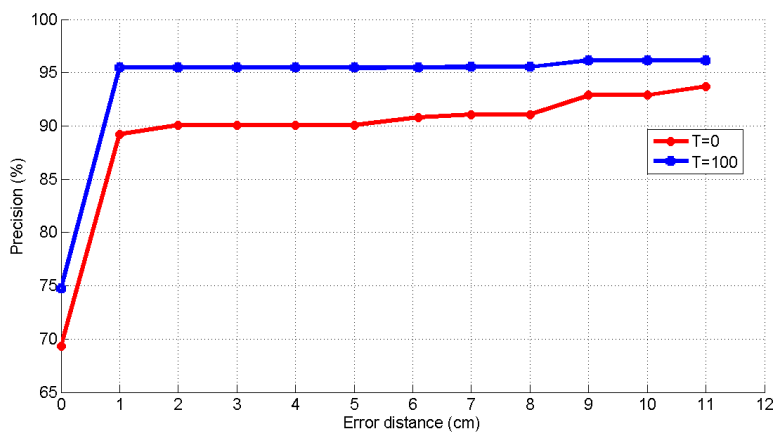

Fig. 8: Precision versus accuracy, $d=1 m, T \in\{0,100\}$

TABLE II: Precision for $1 \mathrm{~cm}$ accuracy, $d \in\{1 \mathrm{~m}, 1.2 \mathrm{~m}, 1.4 \mathrm{~m}\}$

\begin{tabular}{|c||c||c||c|}
\hline $\mathrm{d}(\mathrm{m})$ & 1 & 1.2 & 1.4 \\
\hline Precision for $1 \mathrm{~cm}$ accuracy (\%) & 95 & 90 & 81.5 \\
\hline
\end{tabular}

observe a slight deterioration of respectively $5 \%$ and $13.5 \%$ when increasing $d$ from $1 \mathrm{~m}$ to $1.2 \mathrm{~m}$ and from $1 \mathrm{~m}$ to $1.4 \mathrm{~m}$. However, the system still present good results: An accuracy of $1 \mathrm{~cm}$ within $90 \%$ and $81.5 \%$ precision are obtained for $1.2 \mathrm{~m}$ and $1.4 m$ respectively.

This deterioration is explained by the appearance of pseudopeaks in the low frequency correlation diagram as shown in figure 9 . This may be overcome by readjusting the parameters of the simulated annealing. However, in this case, the receiver may need much time in order to reach the source. Indeed, the simulated annealing algorithm must start at high temperature that allows the receiver to jump out of local minimum. Since the temperature is reduced slowly, the time needed to approach zero temperature will be important. A second solution may consist in using or adding a carrier frequency of $100 \mathrm{~Hz}$ to $200 \mathrm{~Hz}$ and $4 k \mathrm{~Hz}$. Reducing the value of frequency will enlarge the width of the focal zone and induces the disappearance of the pseudo-peaks in the low frequency correlation diagram. This is demonstrated in figure 10 .

On the other hand, we investigate the effect of displacement step on system performance. Increasing the step reduces the time needed for the receiver to reach the source.

As shown in figure 11, performance obtained with a step of $1 \mathrm{~cm}$ is close to that obtained with $5 \mathrm{~cm}$. Deterioration in precision of $15 \%$ for an accuracy of $1 \mathrm{~cm}$ is observed when increasing the step from $1 \mathrm{~cm}$ to $10 \mathrm{~cm}$. However, the system still provides good results.

Next, an experimental evaluation of the system is presented.

\section{B. Experiment results}

1) Experiment protocol: A classic experiment of Time reversal requires an initial source that emit an acoustic signal in the medium. The signals are recieved by the mcirophones of time reversal mirror,then, time reversed. The resulted signals are re-emitted in the medium by the speakers of the 


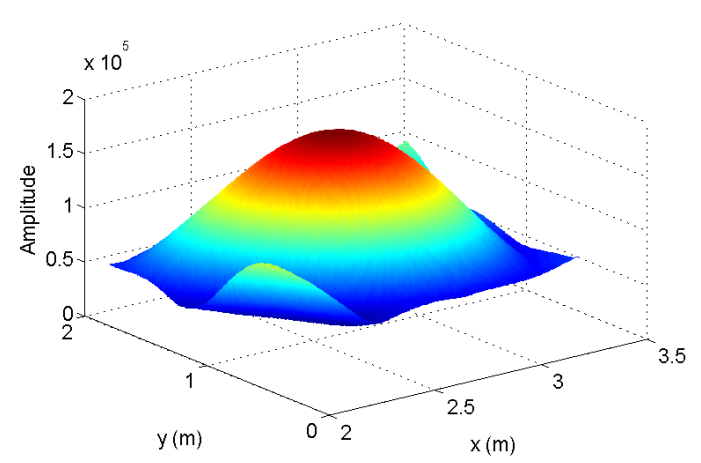

Fig. 9: Appearance of pseudo-peaks in the low frequency spatial correlation diagram, $f=200 \mathrm{~Hz}, d=1.4 \mathrm{~m}$, Source at position $(2.72 m ; 1.14 m ; 1.5 m)$.

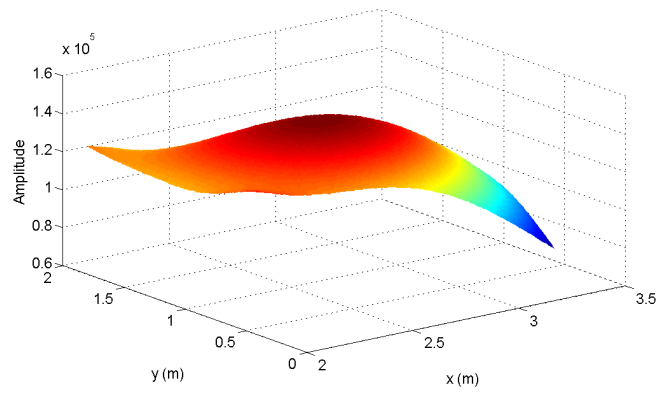

Fig. 10: Low frequency spatial correlation diagram obtained with $f=100 \mathrm{~Hz}, d=1.4 \mathrm{~m}$, Source at position $(2.72 m ; 1.14 m ; 1.5 m)$.

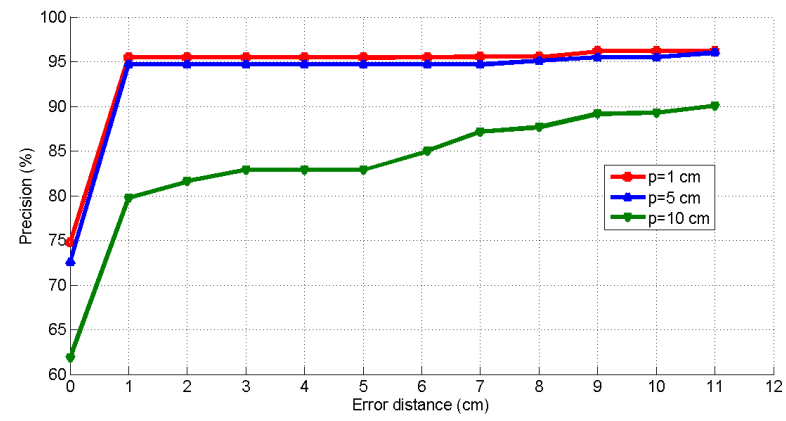

Fig. 11: Precision versus accuracy of the proposed method, $d=1 \mathrm{~m}, T=100, p \in\{1 \mathrm{~cm}, 5 \mathrm{~cm}, 10 \mathrm{~cm}\}$

mirror.

In this experiment, we take advantage of the spatial reciprocity in order to create an initial virtual source [12]. Indeed, instead of emitting a sound with an initial source located at the position $s$ and record the field simultaneously by $N$ microphones of the mirror, the same signal is transmitted successively by each of the speakers of the time reversal mirror, and recorded by the microphone of reference, placed at the position $s$. The recorded signals are time reversed then, emitted simultaneously by the $N$ speakers.

This application of spatial reciprocity theorem then allows to use only one microphone to experience focusing and $N$ speakers, rather than $(2 N+2)$ transducers.

2) Material: The hardware system is composed of a signal acquisition module, an amplifier, four speakers, one microphone and a PC (see figure 12). In the first phase of time reversal, the signal acquisition module deploys one uniform output channel to broadcast successively the signal generated by the PC and, one uniform input channel to record the received signal. The four received signals are then time reversed via MATLAB.

In the second phase, the signal acquisition module deploys four uniform output channels to broadcast simultaneously the time reversed signals. One uniform input channel is used to record the received signal.

Data acquisition between the PC and the signal acquisition module is performed through lab view and signal processing is done via MATLAB.

The described material has been employed to perform measurements in a practical training room in National School of Engineers of Le Mans. The effective area, where we perform the experiment, is shown in figure 13 and measures $4 m \times 4 m \times$ $2.2 \mathrm{~m}$. As in simulations, the four transducers are placed at positions $(0.2 m, 2 m, 2 m),(2 m, 0.2 m, 2 m),(2 m, 3.8 m, 2 m)$ and $(3.8 m, 2 m, 2 m)$ and the system variables were set to:

- $51200 \mathrm{~Hz}$ for sampling frequency,

- $200 \mathrm{~Hz}$ and $4 \mathrm{kHz}$ for low and high carrier frequencies,

- 127chips for length of Gold code sequence,

We choose to localize 2 sources in the effective area, considering that the obtained results can be extrapolated to other sources in the effective area. The first source is placed on the center of the effective area (i.e., at position $(2 m, 2 m, 1.52 m)$ ) and the second is situated at $(2.5 m, 1.5 m, 1.52 m)$. For each source, we have measured using the microphone of reference, with a spatial sample rate of $2 \mathrm{~cm}$, the signal received at points in the square centered on the source and whose side is equal to $48 \mathrm{~cm}$. The square is shown in figure 14 and contains 625 points. 1250 measurements have then been performed.

3) Results: Performance are given, for each source, in terms of the precision versus accuracy plots.

First, we report in figures 15 and 16 the spatial correlation diagrams, obtained for low and high frequencies, for the source at the center of the effective area and the source at the position $(2.5 m, 1.5 m, 1.52 m)$ respectively. As can be observed, the spatial correlation diagrams have similar shape as that found in simulations: For low frequency, the diagram is flat and for high frequency, we observe sharp peak at the source position and pseudo-peaks in its neighborhood.

For the simulated annealing, we have adjusted the initial temperature $T$ to 0.1 and kept the same values as in simulations for the rest of parameters.

Precision versus accuracy for both sources are illustrated in tables III and IV. Both sources are correctly localized within 


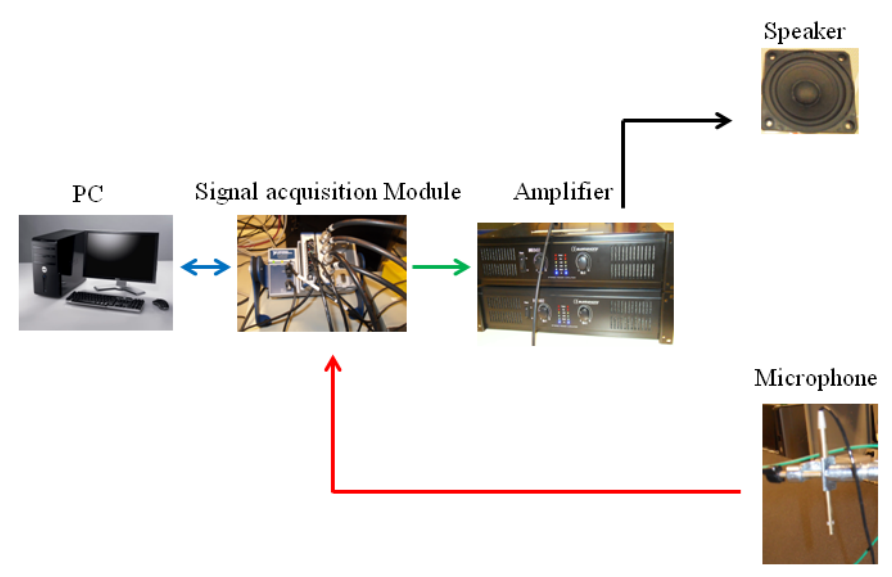

Fig. 12: Material

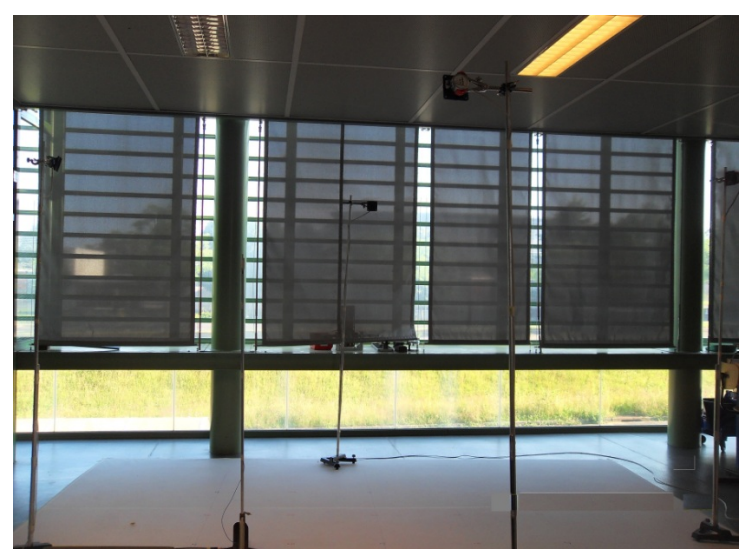

Fig. 13: Experiment environement

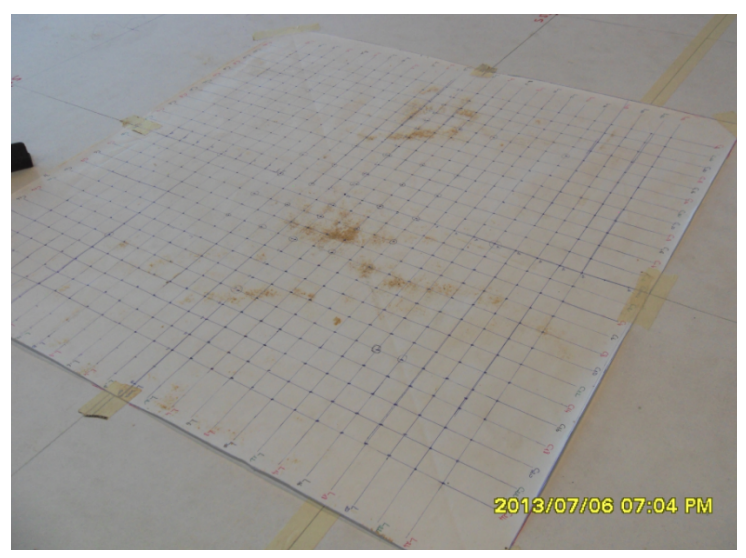

Fig. 14: The measurement square

$96 \%$ precision. We can say that the results are similar to those found simulations.

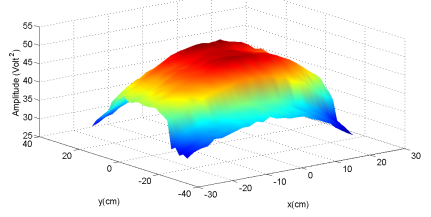

(a) for low frequency

Fig. 15: Spatial correlation $(2 m, 2 m, 1.52 m)$

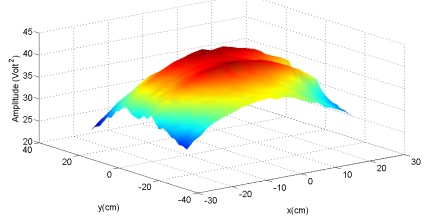

(a) for low frequency

Fig. 16: Spatial correlation diagrams at position $(2.5 m, 1.5 m, 1.52 m)$

TABLE III: Positioning accuracy and precision of source at position $(2 m, 2 m, 1.52 m)$ obtained with $T=0.1$

\begin{tabular}{|c|c|c|c|c|c|c|c|}
\hline Error distance (cm) & 0 & 2 & 4 & 6 & 8 & 10 & 12 \\
\hline Precision(\%)(low frequency) & 0 & 12.8 & 12.8 & 12.8 & 56.8 & 100 & 100 \\
\hline Precision(\%)(proposed method) & 100 & 100 & 100 & 100 & 100 & 100 & 100 \\
\hline
\end{tabular}

TABLE IV: Positioning accuracy and precision of source at position $(2.5 m, 1.5 m, 1.52 m)$ obtained with $T=0.1$

\begin{tabular}{|c|c|c|c|c|c|c|c|}
\hline Error distance (cm) & 0 & 2 & 4 & 6 & 8 & 10 & 12 \\
\hline Precision(\%)(low frequency) & 0 & 0 & 0 & 55 & 96 & 96 & 96 \\
\hline Precision(\%)(proposed method) & 96 & 96 & 96 & 96 & 96 & 96 & 96 \\
\hline
\end{tabular}

\section{CONCLUSION}

This paper has presented an acoustic localization system based on Time reversal. The system has been evaluated by simulations and experiments. The results have shown that the system has subcentimeter accuracy.

We suggest several future directions based on this work. First, we will investigate the possibility of making the system work in a wider space by using lower carrier frequency. Second, we aim to deploy more than two carrier frequencies so that we avoid using the maximum algorithm for high frequency range and reduce the number of the receiver displacement.

\section{ACKNOWLEDGEMENT}

The authors would like to thank M. Mathieu Secail-Geraud for his collaboration. 


\section{REFERENCES}

[1] Daly, P. 1993. Navstar GPS and GLONASS: global satellite navigation system. Electronics and Communication Engineering Journal 349-357.

[2] Bahl, P. and V.N. Padmanabhan. 2000. RADAR: An In-Building RFBased User Location and Tracking System. Proc. IEEE INFOCOM (2):775-784.

[3] Nadia ALOUI, Kosai RAOOF, Ammar BOUALlEGUE , Stephane LETOURNEUR and Sonia ZAIBI, A Novel Indoor Localization Scheme Based On Fingerprinting Technique And CDMA Signals, 2012 International Conference on Indoor Positioning and Indoor Navigation, 13-15th November 2012

[4] Active Bat website, 2008, http://www.cl.cam.ac.uk/research/dtg/attarchive/ bat/

[5] N. Priyantha, A. Chakraborty, and H. Balakrishnan, The Cricket locationsupport system, in Proc. 5th intl. conf. (MobiCom). ACM, 2000, pp. 32-43.

[6] Cem Sertatila, Mustafa A. Altinkayaa, Kosai Raoof, A Novel Acoustic Indoor Localization System Employing CDMA, Digital Signal Processing, vol.22, no.3, (2012), pp.506-517.

[7] Wei-wei Wang, Jing-song Hong, Guang-Min Zhang, Bing-Zhong Wang Node Localization Based on Time Reversal In Wireless Sensor Network, Microwave and Millimeter Wave Technology (ICMMT), 2010.

[8] Alexander Sutin, Yegor Sinelnikov, Time Reversal Acoustic Approach for Non-Lethal Swimmer Deterrent, Waterside Security Conference (WSS), 2010 International, 3-5 Nov. 2010

[9] M.Fink, G.Montaldo, M. Tanter, Time Reversal Acoustics. 2004 IEEE International Ultrasound, Ferroelectrics, and frequency Control Joint 50th Anniversary Conference.

[10] D. Cassereau and M. Fink. Time-reversal of ultrasonic fields. iii. theory of the closed time-reversal cavity. IEEE Trans. Ultra. Ferr. Freq. Cont. 39-5:579592, 1992.

[11] J.B. Allen and D.A Berkley,Image method for efficiently simulating small-room acoustics. J. Acoust. Soc. Am. 65(4), Apr. 1979

[12] Eric BAVU, le puits a retournement temporel dans le domaine audible : un outil de focalisation et dimagerie a haute resolution de sources sonores et vibratoires, Ecole Doctorale : Sciences Mecaniques, Acoustique et Electronique de Paris. 